Samuel A. Wells Jr, National Cancer Institute, National Institutes of Health, Bethesda, MD; Bruce G. Robinson, Kolling Institute of Medical Research, University of Sydney, Sydney, Australia; Robert F. Gagel, University of Texas MD Anderson Cancer Center, Houston, TX; Henning Dralle, Martin Luther University Halle-Wittenberg, Halle, Germany; James A. Fagin, Memoria Sloan-Kettering Cancer Center, New York, NY; Massimo Santoro, Universita' di Napol Federico II, Naples, Italy; Eric Baudin and Martin J. Schlumberger, Institut Gustave Roussy, Villejuif, France; Rossella Elisei, University of Pisa, Pisa, Italy; Barbara Jarzab, Maria Sklodowska-Curie Memorial Cancer Center, Gliwice, Poland; James R. Vasselli and Peter Langmuir, AstraZeneca, Wilmington, DE; Jessica Read, AstraZeneca, Macclesfield; and Anderson J. Ryan, University of Oxford, Oxford, United Kingdom.

Submitted February 18, 2011; accepted July 11, 2011; published online ahead of print at www.jco.org on October 24, 2011

Supported by AstraZeneca and by John Matthew, Mudskipper Bioscience (medical writing support)

Presented at the 46th Annual Meeting of the American Society of Clinical Oncology, Chicago, IL, June 4-8, 2010, and as a poster at the 35th Congress of the European Society for Medical Oncology, Milan, Italy, October 8-12, 2010

Authors' disclosures of potential conflicts of interest and author contributions are found at the end of this article.

Clinical Trials repository link available on JCO.org.

Corresponding author: Samuel A. Wells Jr, MD, Medical Oncology Branch, National Cancer Institute, National Institutes of Health, Building 10, Room 13N-240E MSC 1206, 9000 Rockville Pike, Bethesda, MD 20892; e-mail: wellss@mail.nih.gov.

C 2011 by American Society of Clinical Oncology

0732-183X/12/3002-134/\$20.00

DOI: 10.1200/JCO.2011.35.5040

\title{
Vandetanib in Patients With Locally Advanced or Metastatic Medullary Thyroid Cancer: A Randomized, Double-Blind Phase III Trial
}

Samuel A. Wells Jr, Bruce G. Robinson, Robert F. Gagel, Henning Dralle, James A. Fagin, Massimo Santoro, Eric Baudin, Rossella Elisei, Barbara Jarzab, James R. Vasselli, Jessica Read, Peter Langmuir, Anderson J. Ryan, and Martin J. Schlumberger

See accompanying editorial on page 119 and article on page 200

$$
\begin{array}{llllllll}
\text { A } & \text { B } & \text { S } & \text { T } & \text { R } & \text { A } & \text { C } & \text { T }
\end{array}
$$

\section{Purpose}

There is no effective therapy for patients with advanced medullary thyroid carcinoma (MTC). Vandetanib, a once-daily oral inhibitor of RET kinase, vascular endothelial growth factor receptor, and epidermal growth factor receptor signaling, has previously shown antitumor activity in a phase II study of patients with advanced hereditary MTC.

\section{Patients and Methods}

Patients with advanced MTC were randomly assigned in a 2:1 ratio to receive vandetanib $300 \mathrm{mg} / \mathrm{d}$ or placebo. On objective disease progression, patients could elect to receive open-label vandetanib. The primary end point was progression-free survival (PFS), determined by independent central Response Evaluation Criteria in Solid Tumors (RECIST) assessments.

\section{Results}

Between December 2006 and November 2007, 331 patients (mean age, 52 years; 90\% sporadic; 95\% metastatic) were randomly assigned to receive vandetanib (231) or placebo (100). At data cutoff (July 2009; median follow-up, 24 months), 37\% of patients had progressed and 15\% had died. The study met its primary objective of PFS prolongation with vandetanib versus placebo (hazard ratio [HR], 0.46; $95 \% \mathrm{Cl}, 0.31$ to $0.69 ; P<.001)$. Statistically significant advantages for vandetanib were also seen for objective response rate $(P<.001)$, disease control rate $(P=.001)$, and biochemical response $(P<$ .001). Overall survival data were immature at data cutoff ( $\mathrm{HR}, 0.89 ; 95 \% \mathrm{Cl}, 0.48$ to 1.65$)$. A final survival analysis will take place when $50 \%$ of the patients have died. Common adverse events (any grade) occurred more frequently with vandetanib compared with placebo, including diarrhea (56\% $\vee 26 \%)$, rash $(45 \% \vee 11 \%)$, nausea (33\% v 16\%), hypertension (32\% v 5\%), and headache $(26 \%$ $\checkmark 9 \%)$.

\section{Conclusion}

Vandetanib demonstrated therapeutic efficacy in a phase III trial of patients with advanced MTC (ClinicalTrials.gov NCT00410761).

\section{J Clin Oncol 30:134-141. (C) 2011 by American Society of Clinical Oncology}

\section{INTRODUCTION}

Medullary thyroid carcinoma (MTC), a malignancy of the parafollicular $\mathrm{C}$ cells of the thyroid gland, accounts for approximately $5 \%$ of all thyroid cancers and presents either sporadically $(75 \%$ of patients) or in a hereditary pattern. ${ }^{1,2}$ The 10-year overall survival rate in unselected patients with MTC is approximately $75 \%$, but it decreases to $40 \%$ or less in patients with locally advanced or metastatic disease. ${ }^{1,3,4}$ Neither radiotherapy nor chemotherapy has demonstrated durable objective responses in patients with advanced MTC. ${ }^{5,6}$
Germline mutations in the RET (rearranged during transfection) proto-oncogene occur in virtually all patients with hereditary MTC. ${ }^{7-9}$ Approximately $50 \%$ of patients with sporadic MTC have somatic RET mutations, and $85 \%$ of them have the M918T mutation. ${ }^{10,11}$ Evidence from preclinical studies of molecular targeted therapeutics with activity against RET demonstrate that RET kinase is a potential therapeutic target in MTC. ${ }^{12-14}$ Other signaling pathways likely to contribute to the growth and invasiveness of MTC include vascular endothelial growth factor receptor (VEGFR)-dependent tumor angiogenesis and 
epidermal growth factor receptor (EGFR)-dependent tumor cell proliferation. ${ }^{15}$

Vandetanib is a once-daily oral agent that selectively targets RET, VEGFR, and EGFR signaling. ${ }^{12,16}$ We report the results of an international, randomized, placebo-controlled, double-blind, phase III study (ZETA) to evaluate vandetanib $300 \mathrm{mg} / \mathrm{d}$ in patients with locally advanced or metastatic MTC.

\section{PATIENTS AND METHODS}

\section{Eligibility}

Eligible patients were adults who had measurable, unresectable locally advanced or metastatic, hereditary or sporadic MTC. Submission of a tumor sample was required except for patients with hereditary MTC who had a documented germline RET mutation. Other key inclusion criteria were WHO performance status of 0 to 2 and serum calcitonin level $\geq 500 \mathrm{pg} / \mathrm{mL}$. Exclusion criteria included significant cardiac, hematopoietic, hepatic, or renal dysfunction and administration of chemotherapy and/or radiation therapy within 4 weeks before random assignment. All patients provided written informed consent. The protocol was approved by all relevant institutional ethical committees or review bodies, and the study was conducted in accordance with the Declaration of Helsinki, Good Clinical Practice, and the AstraZeneca policy on bioethics.

\section{Study Design and Treatments}

Patients recruited to this multicenter phase III study were randomly assigned in a 2:1 ratio to receive oral vandetanib at a starting dose of $300 \mathrm{mg} / \mathrm{d}$ or placebo until disease progression. On objective disease progression based on investigator assessment, patients discontinued study treatment, were unblinded, and could elect to enter postprogression, open-label treatment with vandetanib until a withdrawal criterion was met. All patients were to be followed for survival.

The primary objective was to determine whether vandetanib, compared with placebo, prolonged progression-free survival (PFS) on the basis of independent central review. Secondary assessments included objective response rate, disease control rate at 24 weeks, duration of response, overall survival, biochemical response (decreases in serum levels of calcitonin and carcinoembryonic antigen [CEA]), and time to worsening of pain (for time to worsening of pain, see Methods and Results in the Appendix, online only).

The principal investigator in collaboration with the study sponsor, AstraZeneca, designed the clinical trial. The sponsor provided funding and organizational support, collected and managed the data, and performed the statistical analysis. Each author reviewed and approved the manuscript and the principal investigator had final responsibility for the decision to submit for publication. The senior academic authors developed the manuscript, and all coauthors contributed to the manuscript.

\section{Efficacy}

PFS was determined from objective tumor measurements performed at screening and then every 12 weeks until progression or withdrawal of consent.

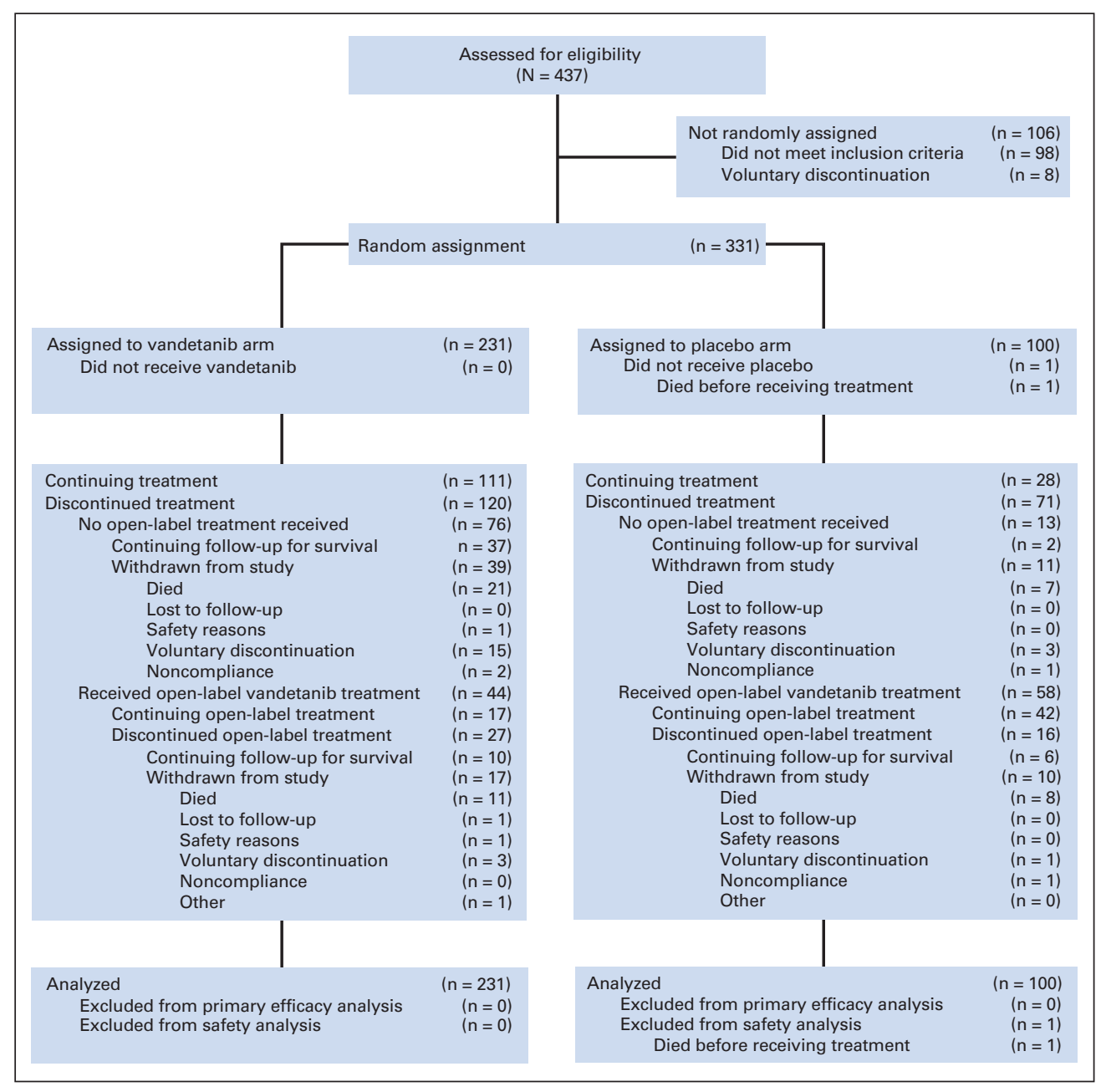

Fig 1. CONSORT diagram 
Tumor assessments were categorized by the investigator by using Response Evaluation Criteria in Solid Tumors v1.0 (RECIST). ${ }^{17}$ Responses were confirmed by central review of separate assessments performed at least 4 weeks apart. RECIST assessments derived from an independent central review of patient scans were the basis for the primary analysis. If, according to central review, progression had not occurred by the time a patient entered open-label treatment, open-label RECIST assessments were also used in the derivation of PFS, objective response rate, disease control rate, and duration of response. PFS was defined from the date of random assignment to the date of objective progression or death (by any cause in the absence of progression within 3 months of the last evaluable RECIST assessment). Patients who had not progressed or who had died at the time of analysis were censored at the time of their last evaluable RECIST assessment.

Overall survival was calculated from the date of random assignment to the date of death with patients followed every 12 weeks until withdrawal of consent or death. Patients who had not died at the time of analysis were censored at the time they were last known to be alive. A final analysis of overall survival is planned when $50 \%$ of the study patients have died.

\section{RET Mutational Status}

The presence of an RET mutation was determined by a combination of two methods: (1) an amplification-refractory mutation system (ARMS) assay that specifically detects the most common RET mutation (M918T) found in sporadic MTC, and (2) direct DNA sequencing following polymerase chain reaction amplification of RET (exons 10, 11, and 13 to 16). A

\begin{tabular}{|c|c|c|c|c|}
\hline \multicolumn{5}{|c|}{$\begin{array}{l}\text { Table 1. Baseline Demographics and Patient Characteristics } \\
\text { (intention-to-treat population; all randomly assigned patients) }\end{array}$} \\
\hline \multirow[b]{2}{*}{ Characteristic } & \multicolumn{2}{|c|}{$\begin{array}{l}\text { Vandetanib } \\
(300 \mathrm{mg}) \\
(\mathrm{n}=231)\end{array}$} & \multicolumn{2}{|c|}{$\begin{array}{l}\text { Placebo } \\
(\mathrm{n}=100)\end{array}$} \\
\hline & No. & $\%$ & No. & $\%$ \\
\hline \multicolumn{5}{|l|}{$\overline{\text { Sex }}$} \\
\hline Male & 134 & 58 & 56 & 56 \\
\hline Female & 97 & 42 & 44 & 44 \\
\hline Mean age, years & \multicolumn{2}{|c|}{50.7} & \multicolumn{2}{|c|}{53.4} \\
\hline \multicolumn{5}{|l|}{ WHO performance status } \\
\hline 0 & 154 & 67 & 58 & 58 \\
\hline 1 & 67 & 29 & 38 & 38 \\
\hline 2 & 10 & 4 & 4 & 4 \\
\hline \multicolumn{5}{|l|}{ Disease type } \\
\hline Hereditary & 28 & 12 & 5 & 5 \\
\hline Sporadic or unknown & 203 & 88 & 95 & 95 \\
\hline Locally advanced & 14 & 6 & 3 & 3 \\
\hline Metastatic & 217 & 94 & 97 & 97 \\
\hline Hepatic & 154 & 67 & 64 & 64 \\
\hline Lymph nodes & 135 & 58 & 68 & 68 \\
\hline Respiratory & 126 & 54 & 60 & 60 \\
\hline Bone/locomotor & 78 & 34 & 40 & 40 \\
\hline Neck & 33 & 14 & 17 & 17 \\
\hline \multicolumn{5}{|l|}{$\begin{array}{l}\text { No. of organs involved } \\
\text { (excluding thyroid) }\end{array}$} \\
\hline 0 or 1 & 29 & 13 & 8 & 8 \\
\hline$\geq 2$ & 202 & 87 & 92 & 92 \\
\hline \multicolumn{5}{|l|}{$\begin{array}{l}\text { Prior systemic therapy for } \\
\quad \text { MTC }\end{array}$} \\
\hline 0 & 141 & 61 & 58 & 58 \\
\hline$\geq 1$ & 90 & 39 & 42 & 42 \\
\hline \multicolumn{5}{|l|}{ RET mutation } \\
\hline Positive & 137 & 59 & 50 & 50 \\
\hline Negative & 2 & 1 & 6 & 6 \\
\hline Unknown & 92 & 40 & 44 & 44 \\
\hline $\begin{array}{l}\text { Abbreviations: MTC, } \\
\text { during transfection. }\end{array}$ & $y$ th & . & $E T$, & \\
\hline
\end{tabular}

mutation-positive sample had either M918T by ARMS assay or an RET mutation in any of exons 10,11 , and 13 to 16 . Conversely, a mutationnegative sample had no M918T mutation by ARMS and a wild-type RET sequence in each of exons 10,11, and 13 to 16 . The mutation status was declared unknown in cases in which an assay failed to yield a sequence at any of the tested exons (by sequencing or ARMS assay), and none of the successful assays demonstrated a mutation.

\section{Measurement of Serum Tumor Markers}

Blood samples for calcitonin and CEA analysis were collected at baseline (day 1), every 4 weeks until week 12 , and then every 12 weeks thereafter. Serum levels of calcitonin and CEA were determined as previously described. ${ }^{18} \mathrm{~A}$ patient's best biochemical response for either calcitonin or CEA was defined as follows: complete response, normalization of serum levels following treatment confirmed a minimum of 4 weeks later; partial response, $\geq 50 \%$ decrease from baseline levels maintained over a minimum of 4 weeks; stable disease, between $+50 \%$ and $-50 \%$ change from baseline levels maintained for at least 4 weeks; and progressive disease, $\geq 50 \%$ increase from baseline maintained for at least 4 weeks.

\section{Safety and Tolerability}

Safety was assessed throughout the study by monitoring and recording adverse events, 12-lead ECG parameters, vital signs, clinical chemistry, hematology, and urinalysis. Adverse events were assessed by using the National Cancer Institute's Common Terminology Criteria for Adverse Events (CTCAE, v3). Scheduled 12-lead ECGs were performed during screening, at 1, $2,4,8$, and 12 weeks and every 3 months thereafter. The QTc interval was evaluated centrally, and prolongation was defined as a single measurement of $\geq 550 \mathrm{~ms}$ or an increase of $\geq 100 \mathrm{~ms}$ from baseline, two consecutive measurements (within 48 hours of each other) that were $\geq 500 \mathrm{~ms}$ but less than $550 \mathrm{~ms}$, or an increase of $\geq 60 \mathrm{~ms}$ but less than $100 \mathrm{~ms}$ from baseline to a value $\geq 480 \mathrm{~ms}$. Specific dose reduction plans were in place for management of skin toxicity, GI toxicity, and QTc prolongation. There was also a general dose reduction scheme for any CTCAE grade 3 or 4 adverse event (patients started at vandetanib $300 \mathrm{mg} / \mathrm{d}$ or placebo, and dose was reduced to $200 \mathrm{mg} / \mathrm{d}$ for a grade 3 or 4 adverse event; if further grade 3 or 4 toxicity occurred, reduction to $100 \mathrm{mg} / \mathrm{d}$ was allowed).

\section{Statistical Analysis}

The study was designed to have more than $80 \%$ power to detect a hazard ratio (HR) less than 0.50 at a $5 \%$ significance level; a minimum of 90 progression events were required, assuming a median PFS of 12 months in the placebo group and an overall sample size of 232 patients. Analyses of PFS and overall survival were conducted by using the log-rank test (unadjusted model with treatment factor only) in the intention-to-treat population. A sensitivity analysis of PFS was performed by using Cox's proportional hazards regression model, which allowed for the effect of treatment and included terms for RET mutation status, MTC status (hereditary or sporadic), prerandomization historic calcitonin and CEA changes, number of prior therapies, and response to prior therapy. ${ }^{19}$ The following predefined sensitivity analyses were also performed for PFS: per protocol that excluded significant protocol deviators; Whitehead method to assess the impact of a differential frequency of assessments in the two treatment arms; randomized phase alone (ie, excluding the open-label phase); and PFS derived from investigator assessments. ${ }^{20}$ The objective response rate and disease control rate were analyzed by using logistic regression (these variables included open-label assessments). All $P$ values were two-sided. Subgroup analyses of PFS by prespecified baseline characteristics and ad hoc subgroup analyses of PFS and objective response rate by RET mutation status and M918T mutation status were performed.

\section{RESULTS}

\section{Patients}

Between December 7, 2006, and November 21, 2007, 331 patients recruited from 23 countries were randomly assigned to vandetanib (231) or placebo (100; Fig 1). Although not an exact 2:1 


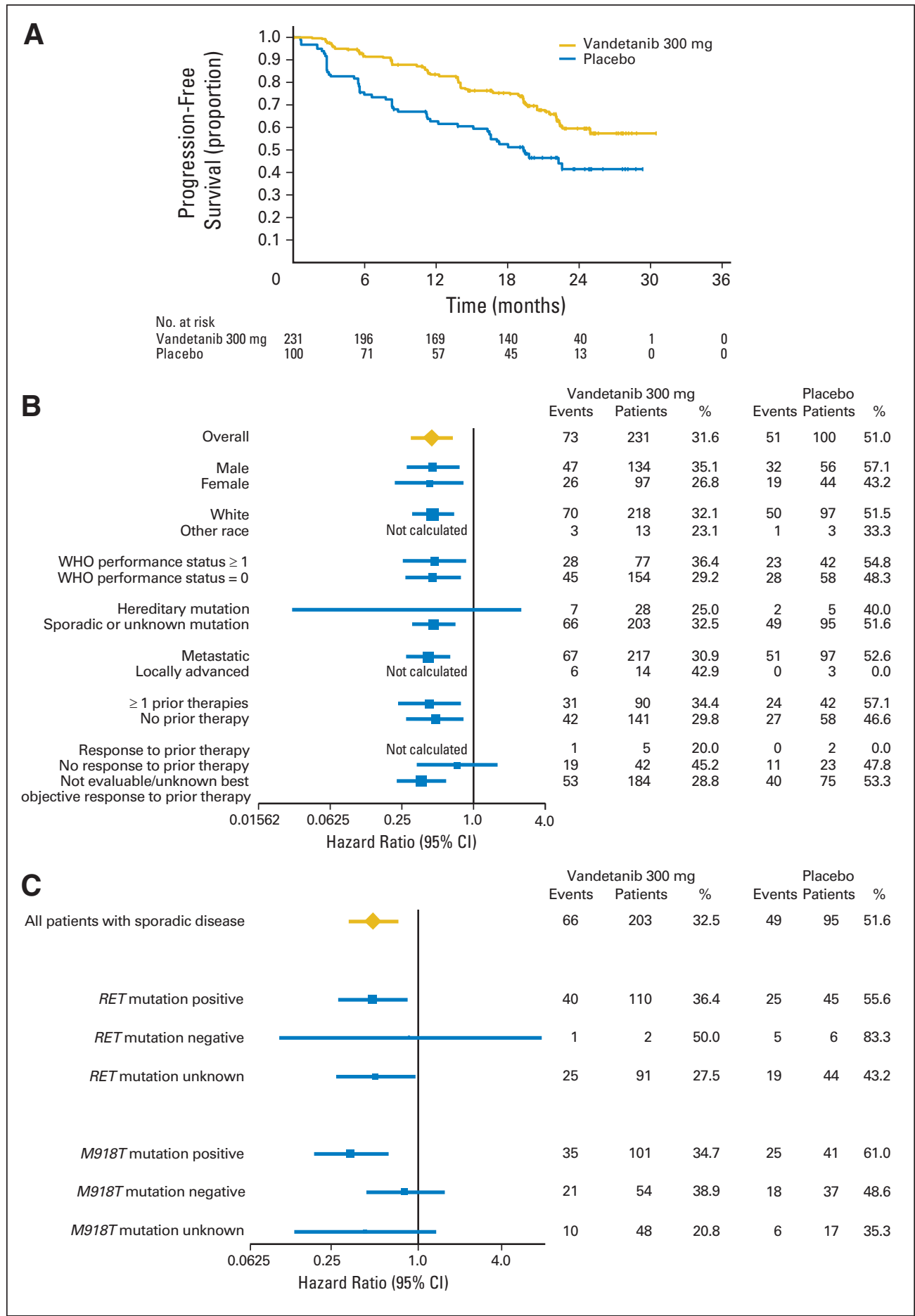

Fig 2. (A) Kaplan-Meier curve of progression-free survival (PFS; intention-to-treat population; all randomly assigned patients); derived from all available centralized Response Evaluation Criteria in Solid Tumors (RECIST) assessments. (B) Forest plot of hazard ratios for PFS according to baseline characteristics and disease status. (C) Forest plot of hazard ratios for PFS according to rearranged during transfection (RET) mutation status and M918T mutation status in patients with sporadic medullary thyroid carcinoma. (B, C) A hazard ratio $<1$ favors vandetanib. The analyses were performed using a log-rank test with treatment as the only factor.

randomization, this imbalance occurred by chance. Patient characteristics and baseline demographics were similar in both arms (Table 1). The majority of patients presented with sporadic disease, and most had metastatic disease at study entry. At data cutoff (July 31, 2009), the median duration of follow-up was 24 months, and 139 patients were continuing blinded treatment: 111 (48\%) randomly assigned to vandetanib and 28 (28\%) randomly assigned to placebo. Among 123 patients who developed tumor progression and were eligible to receive open-label treatment, 93 (vandetanib, 41 of 67 [61\%]; placebo, 52 of 56 [93\%]) elected to enter postprogression open-label treatment with 
vandetanib. Overall, 48 deaths (32, vandetanib arm; 16, placebo arm) occurred at the time of data cutoff, including one patient randomly assigned to the placebo arm who died of progressive MTC before receiving study treatment and who was not included in the safety analysis population. All patients were included in the efficacy analysis.

\section{Efficacy}

At the time of analysis, 124 patients (37\%) had progressed and 48 (15\%) had died. Significant prolongation of PFS was observed for patients receiving vandetanib compared with placebo (HR, 0.46; 95\% CI, 0.31 to $0.69 ; P<.001$; Fig 2A; Table 2). The median PFS was 19.3 months in the placebo group and, although the median had not yet been reached for the vandetanib group, fitting a Weibull model indicated a predicted median of 30.5 months. ${ }^{21}$ The PFS at 6 months was 83\% (vandetanib) and 63\% (placebo). The Kaplan-Meier plot indicates that the relative hazards were larger at earlier time points. In addition to the primary analysis, Cox regression analysis as well as other sensitivity analyses detected an improvement in PFS with vandetanib versus placebo (Table 2 ). A total of 51 patients (23 vandetanib, 28 placebo) received open-label vandetanib before progression by central read was documented. Both visual inspection of the forest plot and the finding of a lack of statistical significance for the planned global interaction test $(P=.177)$ suggest that the PFS benefits observed were generally consistent across all prespecified subgroups (Fig 2B).

Vandetanib also showed significant advantages compared with placebo in the secondary efficacy end points of objective response rate, disease control rate, and calcitonin and CEA biochemical response rates (Table 2). Objective responses were durable on the basis of the median duration of response not being reached at 24 months of follow-up (fitting a Weibull model gives a predicted median duration of response of 22 months). It is important to note that 12 of 13 responses observed in patients initially randomly assigned to placebo occurred while the patients were subsequently receiving vandetanib in the open-label phase. Overall survival data were immature at data cutoff (HR, 0.89; 95\% CI, 0.48 to 1.65 ; Fig 3 ). A final survival analysis will take place when $50 \%$ of the patients have died.

Of the 33 patients with hereditary MTC, 32 had a documented RET germline mutation before study entry, and of the 28 receiving vandetanib, 13 (46.4\%) had an objective response. Paraffin blocks or slides were available for analysis from 297 of 298 patients with sporadic MTC. An RET mutation was present in 155 patients (52.0\%), no RET mutation was present in eight patients $(2.7 \%)$, and the RET mutation status was unknown in 135 patients (45.3\%). There was a high number of patients with unknown RET mutation status because their paraffin blocks or slides had an insufficient quantity or quality of DNA for complete analysis. The small number of RET-negative patients means that subgroup analyses of PFS (Fig 3B) and objective response rate (Table 3 ) by $R E T$ mutation status are inconclusive. In patients with sporadic MTC, however, a subgroup analysis of PFS by M918T mutation suggested that M918T mutation-positive patients had a higher response rate to vandetanib compared with M918T mutationnegative patients (Fig 2C; Table 3).

\section{Safety and Tolerability}

The median duration of treatment in the randomized phase was 90.1 weeks (vandetanib) and 39.9 weeks (placebo). Common adverse events (any grade and grade 3 or higher) are summarized in Table 4. Thirty-one patients discontinued treatment during the randomized phase because of an adverse event: 28 (12\%) receiving vandetanib and three $(3 \%)$ receiving placebo. Adverse events such as diarrhea, rash, nausea, and hypertension occurred in more than $30 \%$ of patients receiving vandetanib; adverse events leading to discontinuation of vandetanib reported in more than $1 \%$ of patients were asthenia $(1.7 \%)$

\begin{tabular}{|c|c|c|c|c|c|c|c|c|}
\hline \multirow[b]{2}{*}{ Progression-Free Survival } & \multicolumn{2}{|c|}{ Vandetanib } & \multicolumn{2}{|l|}{ Placebo } & \multirow[b]{2}{*}{$\mathrm{HR}$} & \multirow[b]{2}{*}{ OR } & \multirow[b]{2}{*}{$95 \% \mathrm{Cl}$} & \multirow[b]{2}{*}{$P$} \\
\hline & $\begin{array}{l}\text { No. of Events/ } \\
\text { No. of Patients }\end{array}$ & $\%$ & $\begin{array}{l}\text { No. of Events/ } \\
\text { No. of Patients }\end{array}$ & $\%$ & & & & \\
\hline Primary analysis & $73 / 231$ & & $51 / 100$ & & 0.46 & & 0.31 to 0.69 & $<.001$ \\
\hline \multicolumn{9}{|l|}{ Predefined sensitivity analyses } \\
\hline Cox proportional hazards model & $73 / 231$ & & $51 / 100$ & & 0.46 & & 0.32 to 0.68 & $<.001$ \\
\hline Per protocol analysis & $71 / 215$ & & $48 / 91$ & & 0.45 & & 0.30 to 0.68 & $<.001$ \\
\hline Whitehead's method & $73 / 231$ & & $51 / 100$ & & 0.51 & & 0.35 to 0.72 & $<.001$ \\
\hline Excluding data from open-label phase & $64 / 231$ & & $59 / 100$ & & 0.27 & & 0.18 to 0.41 & $<.001$ \\
\hline Investigator RECIST assessments & $101 / 231$ & & $62 / 100$ & & 0.40 & & 0.27 to 0.58 & $<.001$ \\
\hline \multicolumn{9}{|l|}{ Secondary efficacy end points } \\
\hline Objective response rate & & 45 & & 13 & & 5.48 & 2.99 to 10.79 & $<.001$ \\
\hline Disease control rate & & 87 & & 71 & & 2.64 & 1.48 to 4.69 & .001 \\
\hline Calcitonin biochemical response rate & & 69 & & 3 & & 72.9 & 26.2 to 303.2 & $<.001$ \\
\hline CEA biochemical response rate & & 52 & & 2 & & 52.0 & 16.0 to 320.3 & $<.001$ \\
\hline \multicolumn{9}{|c|}{ 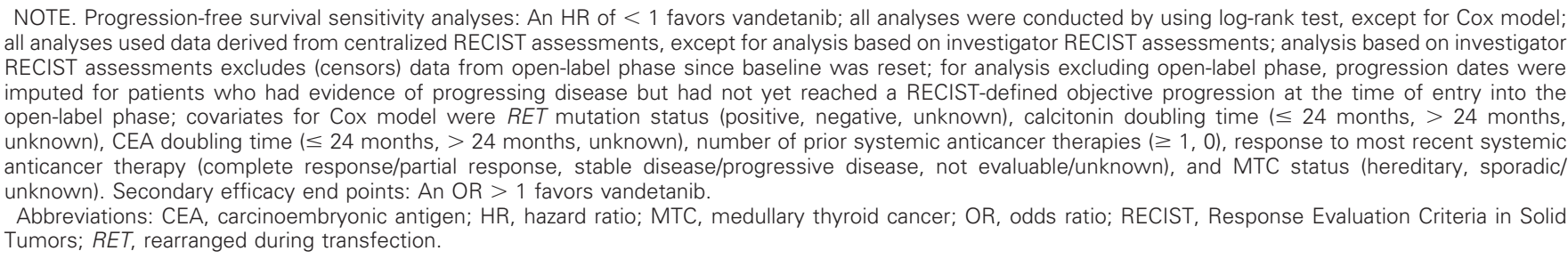 } \\
\hline
\end{tabular}




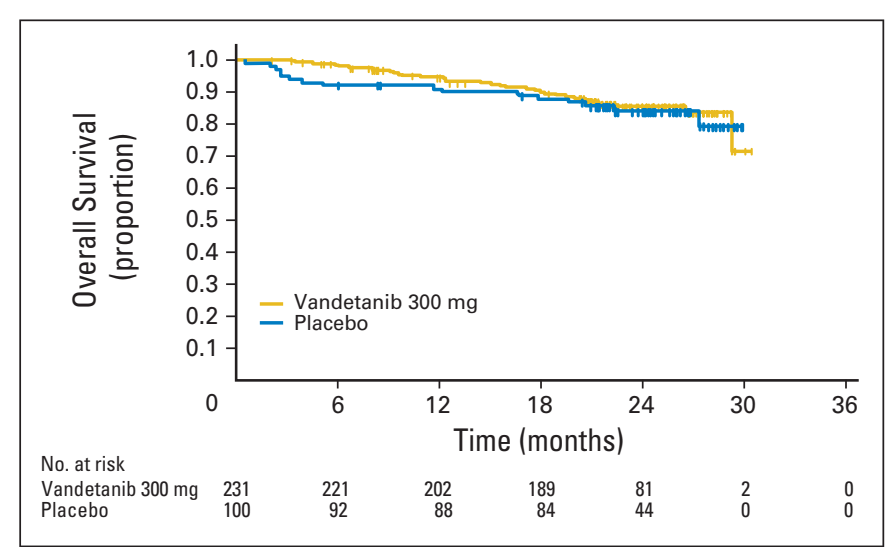

Fig 3. Kaplan-Meier curve of overall survival (intention-to-treat population; all randomly assigned patients).

and rash (1.3\%). More patients required dose reduction of vandetanib compared with placebo for adverse events or QTc prolongation $(35 \%$ v 3\%). Nineteen patients (8\%) developed protocol-defined QTc prolongation, but there were no reports of torsades de pointes. From entry, more patients on vandetanib compared with placebo were noted to have rising thyroid-stimulating hormone serum levels, and they required an increase in thyroid replacement (49.3\% v 17.2\%). Five patients on the vandetanib arm experienced adverse events leading to death during the randomized phase; these were single instances of aspiration pneumonia, respiratory arrest, respiratory failure, staphylococcal sepsis, and arrhythmia and acute cardiac failure in one patient. The two deaths due to an adverse event in the placebo arm were isolated cases of gastroenteritis and GI hemorrhage.

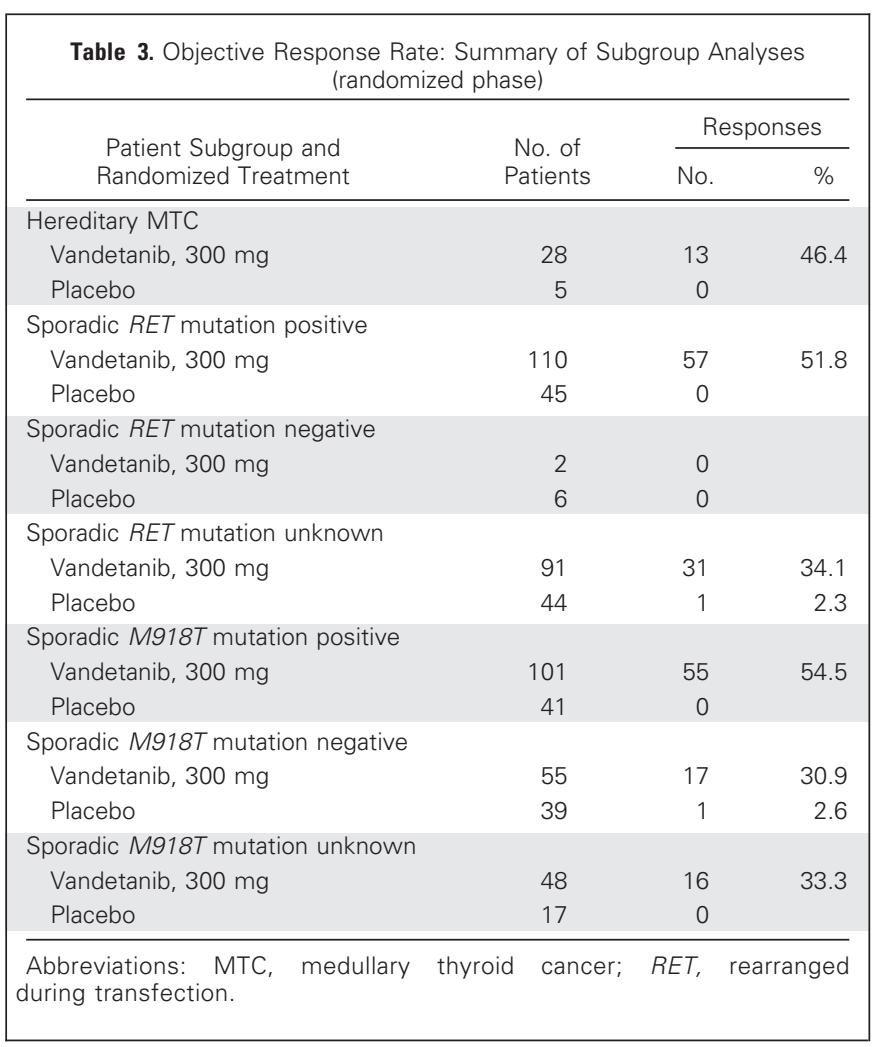

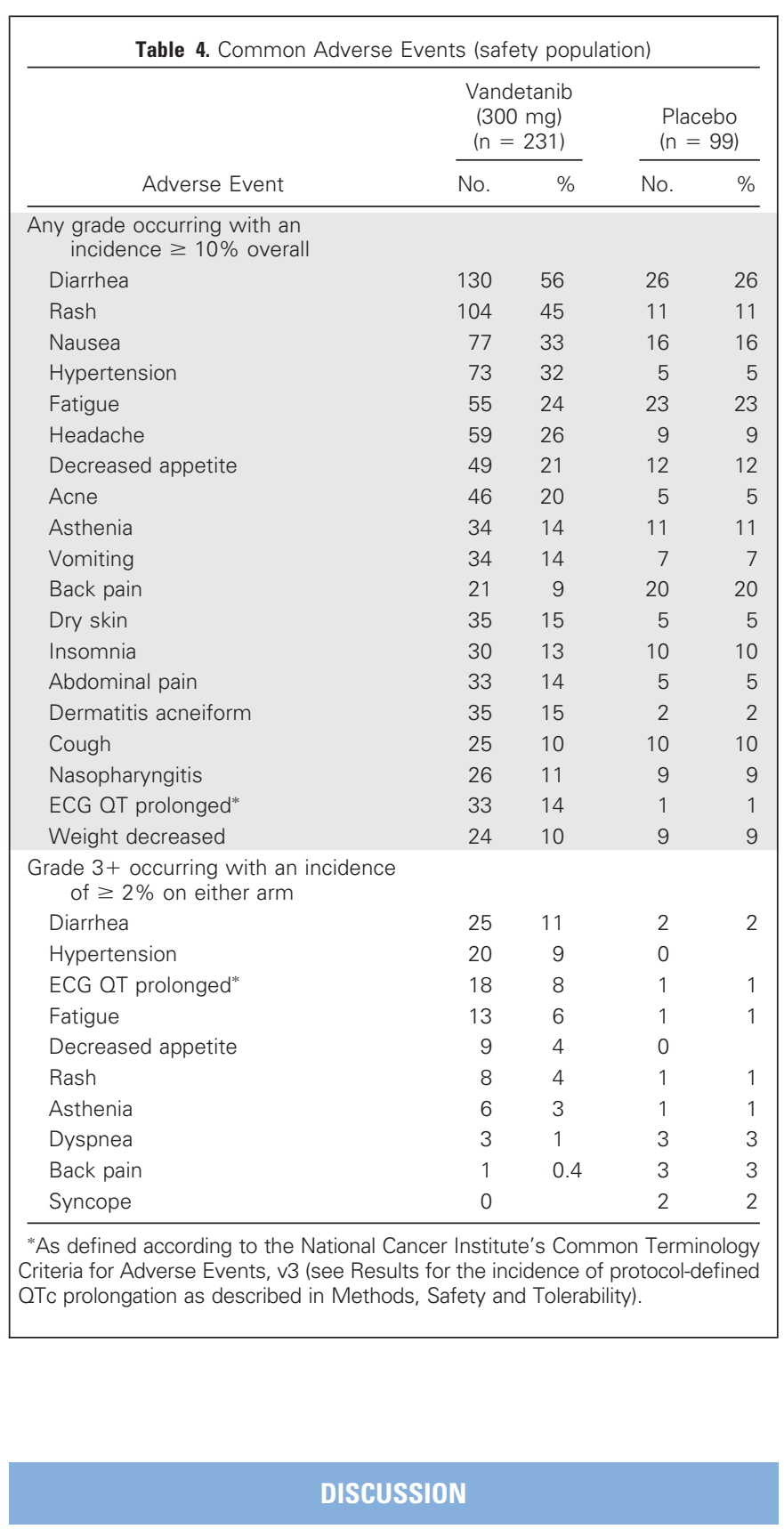

Patients with locally advanced or metastatic MTC are incurable, and chemotherapy and radiation therapy have been largely ineffective. Therefore, the ability to substantially prolong the time to disease progression would benefit such patients. Mutations in the RET protooncogene are central to the development of MTC in virtually all patients with hereditary MTC and in approximately half the patients with sporadic MTC. ${ }^{7-9,11}$ Following preclinical studies demonstrating that vandetanib inhibited signaling through RET kinase, ${ }^{12,22}$ a phase II clinical trial of oral vandetanib $(300 \mathrm{mg}$ ) was initiated in patients with locally advanced or metastatic hereditary MTC. There were confirmed partial remissions in $20 \%$ of patients and stable disease of more than 24 weeks in $73 \%$ of patients. ${ }^{18}$ Published reports of early-phase clinical trials of other tyrosine kinase inhibitors in patients with advanced MTC have shown partial remission rates ranging from $0 \%$ to $25 \%$ in small single-arm trials. ${ }^{23-29}$ 
In this study, there was a significant prolongation of PFS in patients who received vandetanib compared with placebo, with an $H R$ of 0.46 and an estimated 11-month prolongation of median PFS. In addition to the primary analysis, an improvement was also detected in all other predefined sensitivity analyses of PFS, including an HR of 0.27 if open-label scans were excluded. The secondary efficacy end points of objective response rate, disease control rate, and biochemical response also showed statistically significant benefit in the treatment group compared with the control group. Of the 13 objective responses in patients randomly assigned to placebo, 12 occurred while patients were receiving open-label vandetanib. Overall survival data are immature, and the final assessment will occur when $50 \%$ of patients have died; however, the analysis is likely to be confounded by the ability of patients randomly assigned to placebo to receive subsequent treatment with open-label vandetanib.

The benefit that was demonstrated in PFS for patients receiving vandetanib compared with placebo was observed in patients with the hereditary or the sporadic form of MTC. Because of the small number of patients with sporadic MTC who were RET negative and the large number of patients who were RET unknown, the subgroup analyses of PFS and objective response rate by RET mutation status are inconclusive. If data from the ARMS assay are taken into account, patients with sporadic MTC received benefit from vandetanib whether their tumors were $M 918 T$ positive or negative; however, the response rate was greater in those who had an M918T mutation.

Treatment with vandetanib was generally well tolerated. The majority of adverse events were manageable according to standard clinical practice alone or in combination with vandetanib dose reductions, which allowed patients to continue receiving vandetanib treatment for extended periods of time. The rate of vandetanib treatment discontinuation because of toxicity was low (12\%), despite a median duration of treatment of approximately 1 year and 9 months (90.1 weeks).

In this clinical study, vandetanib has shown efficacy in patients with locally advanced or metastatic MTC, a challenging group of patients for whom there has been no effective therapy.

\section{AUTHORS' DISCLOSURES OF POTENTIAL CONFLICTS} OF INTEREST

Although all authors completed the disclosure declaration, the following author(s) indicated a financial or other interest that is relevant to the subject matter under consideration in this article. Certain relationships marked with a "U" are those for which no compensation was received; those relationships marked with a "C" were compensated. For a detailed description of the disclosure categories, or for more information about ASCO's conflict of interest policy, please refer to the Author Disclosure Declaration and the Disclosures of Potential Conflicts of Interest section in Information for Contributors.

Employment or Leadership Position: James R. Vasselli, AstraZeneca (C); Jessica Read, AstraZeneca (C); Peter Langmuir, AstraZeneca (C) Consultant or Advisory Role: Samuel A. Wells Jr, AstraZeneca (C); Bruce G. Robinson, AstraZeneca (C); Robert F. Gagel, AstraZeneca (C); Barbara Jarzab, AstraZeneca (C); Anderson J. Ryan, AstraZeneca (U); Martin J. Schlumberger, AstraZeneca (C) Stock Ownership: James R. Vasselli, AstraZeneca; Anderson J. Ryan, AstraZeneca Honoraria: Robert F. Gagel, AstreaZeneca; James A. Fagin, AstraZeneca; Barbara Jarzab, Exelixis, Eisai Research Funding: Robert F. Gagel, AstraZeneca; Massimo Santoro, AstraZeneca; Barbara Jarzab, AstraZeneca; Martin J. Schlumberger, AstraZeneca Expert Testimony: Robert F. Gagel, AstraZeneca (U); Anderson J. Ryan, AstraZeneca (C) Other Remuneration: Samuel A. Wells Jr, AstraZeneca

\section{AUTHOR CONTRIBUTIONS}

Conception and design: Samuel A. Wells Jr, Bruce G. Robinson, Robert F. Gagel, James A. Fagin, Massimo Santoro, Peter Langmuir, Anderson J. Ryan, Martin J. Schlumberger

Collection and assembly of data: Samuel A. Wells Jr, Bruce G.

Robinson, Robert F. Gagel, Henning Dralle, Eric Baudin, Rossella Elisei, Barbara Jarzab, James R. Vasselli, Peter Langmuir, Anderson J. Ryan, Martin J. Schlumberger

Data analysis and interpretation: Samuel A. Wells Jr, Bruce G. Robinson, Robert F. Gagel, James A. Fagin, James R. Vasselli, Jessica Read, Peter Langmuir, Anderson J. Ryan, Martin J. Schlumberger

Manuscript writing: All authors

Final approval of manuscript: All authors

\section{REFERENCES}

1. Hundahl SA, Fleming ID, Fremgen AM, et al: A National Cancer Data Base report on 53,856 cases of thyroid carcinoma treated in the U.S., 1985-1995 [see comments]. Cancer 83:26382648, 1998

2. Lakhani VT, You YN, Wells SA: The multiple endocrine neoplasia syndromes. Annu Rev Med 58:253-265, 2007

3. Roman S, Lin R, Sosa JA: Prognosis of medullary thyroid carcinoma: Demographic, clinical, and pathologic predictors of survival in 1252 cases. Cancer 107:2134-2142, 2006

4. Modigliani E, Cohen R, Campos JM, et al: Prognostic factors for survival and for biochemical cure in medullary thyroid carcinoma: Results in 899 patients-The GETC Study Group, Groupe d'étude des tumeurs à calcitonine. Clin Endocrinol (Oxf) 48:265-273, 1998

5. Martins RG, Rajendran JG, Capell $P$, et al: Medullary thyroid cancer: Options for systemic therapy of metastatic disease? J Clin Oncol 24:1653-1655, 2006
6. American Thyroid Association Guidelines Task Force, Kloos RT, Eng C, et al: Medullary thyroid cancer: Management guidelines of the American Thyroid Association. Thyroid 19:565-612, 2009

7. Donis-Keller H, Dou S, Chi D, et al: Mutations in the RET proto-oncogene are associated with MEN 2A and FMTC. Hum Mol Genet 2:851-856, 1993

8. Mulligan LM, Kwok JB, Healey CS, et al: Germline mutations of the RET proto-oncogene in multiple endocrine neoplasia type 2A. Nature 363:458-460, 1993

9. Carlson KM, Dou S, Chi D, et al: Single missense mutation in the tyrosine kinase catalytic domain of the RET protooncogene is associated with multiple endocrine neoplasia type 2B. Proc Natl Acad Sci U S A 91:1579-1583, 1994

10. Marsh DJ, Learoyd DL, Andrew SD, et al: Somatic mutations in the RET proto-oncogene in sporadic medullary thyroid carcinoma. Clin Endocrinol (Oxf) 44:249-257, 1996

11. Elisei R, Cosci $B$, Romei $C$, et al: Prognostic significance of somatic RET oncogene mutations in sporadic medullary thyroid cancer: A 10-year follow-up study. J Clin Endocrinol Metab 93:682687, 2008
12. Carlomagno $F$, Vitagliano D, Guida $T$, et al: ZD6474, an orally available inhibitor of KDR tyrosine kinase activity, efficiently blocks oncogenic RET kinases. Cancer Res 62:7284-7290, 2002

13. Carlomagno F, Anaganti S, Guida T, et al: BAY 43-9006 inhibition of oncogenic RET mutants. J Natl Cancer Inst 98:326-334, 2006

14. Santoro M, Carlomagno F: Drug insight: Small molecule inhibitors of protein kinases in the treatment of thyroid cancer. Nat Clin Pract Endocrinol Metab 2:42-52, 2006

15. Rodríguez-Antona C, Pallares J, MonteroConde $\mathrm{C}$, et al: Overexpression and activation of EGFR and VEGFR2 in medullary thyroid carcinomas is related to metastasis. Endocr Relat Cancer 17:716,2010

16. Wedge SR, Ogilvie DJ, Dukes $M$, et al: ZD6474 inhibits vascular endothelial growth factor signaling, angiogenesis, and tumor growth following oral administration. Cancer Res 62:4645-4655, 2002

17. Therasse $P$, Arbuck SG, Eisenhauer EA, et al: New guidelines to evaluate the response to treatment in solid tumors: European Organization for Research and Treatment of Cancer, National Cancer 
Institute of the United States, National Cancer Institute of Canada. J Natl Cancer Inst 92:205-216, 2000

18. Wells SA Jr, Gosnell JE, Gagel RF, et al: Vandetanib for the treatment of patients with locally advanced or metastatic hereditary medullary thyroid cancer. J Clin Oncol 28:767-772, 2010

19. Cox DR: Regression models and life tables. J R Stat Soc B 34:187-220, 1972

20. Whitehead J: The analysis of relapse clinical trials, with application to a comparison of two ulcer treatments. Stat Med 8:1439-1454, 1989

21. Weibull W: A statistical distribution function of wide applicability. J Appl Mech Trans 18:293-297, 1951

22. Vidal M, Wells S, Ryan A, et al: ZD6474 suppresses oncogenic RET isoforms in a Drosophila model for type 2 multiple endocrine neoplasia syndromes and papillary thyroid carcinoma. Cancer Res 65:3538-3541, 2005

23. de Groot JW, Zonnenberg BA, van UffordMannesse $P Q$, et al: A Phase II trial of imatinib therapy for metastatic medullary thyroid carcinoma. $\mathrm{J}$ Clin Endocrinol Metab 92:3466-3469, 2007

24. Frank-Raue K, Fabel M, Delorme $S$, et al: Efficacy of imatinib mesylate in advanced medullary thyroid carcinoma. Eur J Endocrinol 157:215-220, 2007

25. Cohen EE, Rosen LS, Vokes EE, et al: Axitinib is an active treatment for all histologic subtypes of advanced thyroid cancer: Results from a Phase II study. J Clin Oncol 26:4708-4713, 2008
26. Gupta-Abramson V, Troxel AB, Nellore A, et al: Phase II trial of sorafenib in advanced thyroid cancer. J Clin Oncol 26:4714-4719, 2008

27. Pennell NA, Daniels GH, Haddad RI, et al: A Phase II study of gefitinib in patients with advanced thyroid cancer. Thyroid 18:317-323, 2008

28. Schlumberger MJ, Elisei $R$, Bastholt $L$, et al: Phase II study of safety and efficacy of motesanib in patients with progressive or symptomatic, advanced or metastatic medullary thyroid cancer. J Clin Oncol 27:3794-3801, 2009

29. Lam ET, Ringel MD, Kloos RT, et al: Phase Il clinical trial of sorafenib in metastatic medullary thyroid cancer. J Clin Oncol 28:2323-2330, 2010

\section{ATTEND THE 2012 GASTROINTESTINAL CANCERS SYMPOSIUM}

The 2012 Gastrointestinal Cancers Symposium (January 19-21, 2012, in San Francisco, California) is a high-profile, specialized, multidisciplinary oncology event. This Symposium is designed to offer attendees the opportunity to discuss the latest translational and clinical research in the field of gastrointestinal cancers. New topics on this year's program will explore broad issues that affect all gastrointestinal disease sites, such as epidemiology and development of clinical trials. Bookmark gicasym.org and visit regularly for meeting updates. The abstract submitter, registration, and housing selection features are now open.

For more information, please visit gicasym.org/2011.

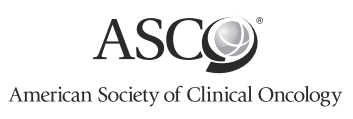

